\title{
A characterization of Commutative Semigroups
}

\author{
Dr. D. Mrudula Devi ${ }^{a}$, Dr. G. Shobha Latha ${ }^{b}$, Dr. K.Vijaya Prasamsa ${ }^{c}$ \\ ${ }^{\text {a} P r o f e s s o r, ~ V i g n a n ~ I n s t i t u t e ~ o f ~ I n f o r m a t i o n ~ a n d ~ T e c h n o l o g y, ~ V i s a k h a p a t n a m ~}$ \\ ${ }^{\mathrm{b}}$ Professor, S.K. UNIVERSITY ANANTHAUR \\ ${ }^{\mathrm{c}}$ Associate Professor, Vignan Institute of Information and Technology, Visakhapatnam \\ amruduladevisai@gmail.com, b.shobhalatha@yahoo.com, ${ }^{c}$ kvprasamsa@gmail.com
}

Article History: Received: 10 November 2020; Revised 12 January 2021 Accepted: 27 January 2021; Published online: 5 April 2021

\begin{abstract}
This paper deals with some results on commutative semigroups. We consider (s,.) is externally commutative right zero semigroup is regular if it is intra regular and (s,.) is externally commutative semigroup then every inverse semigroup is $\mathrm{u}$ - inverse semigroup. We will also prove that if $(\mathrm{S},$.$) is a \mathrm{H}$ - semigroup then weakly cancellative laws hold in $\mathrm{H}$ - semigroup. In one case we will take $(\mathrm{S},$.$) is commutative left regular semi group and we will prove that (\mathrm{S},$.$) is \Pi$ - inverse semigroup. We will also consider $(\mathrm{S},$.$) is commutative weakly balanced semigroup and then prove every left (right) regular semigroup is$ weakly separate, quasi separate and separate. Additionally, if $(\mathrm{S},$.$) is completely regular semigroup we will prove that (\mathrm{S},$.$) is$ permutable and weakly separtive. One a conclusing note we will show and prove some theorems related to permutable semigroups and GC commutative Semigroups..

Keywords: Intra regular, H - semigroup, Inverse semigroup, Quasi separate, weakly separate, Permutable, Completely Regular semigroup, П - Regular.
\end{abstract}

\section{Introduction}

Research on commutative semigroup has a long history. Lawson (1996) made a good case that the earliest article which would currently receive a classification in an 1826 paper by Abel which clearly contains cancellative commutative semigroups. A. semigroup $\mathrm{s}$ is commutative if the defining binary operation is commutative. That is for all $\mathrm{X}, \mathrm{Y}$ in $\mathrm{S}$ the identity $\mathrm{x} . \mathrm{y}=\mathrm{y} \cdot \mathrm{x}$ holds. Although the term Abelian semigroup is sometimes used, it is more commonly referred as commutative semigroups. In this paper we present the results on Commutative semigroups. The motivation to prove the theorems is due to the results of J.M. HOWIE [1], P. SRINIVASULA REDDY and G. SHOBHA LATHA [2] Tamura, T. and Kimura, N [3]

\section{Preliminaries}

1.1.Definition: A semigroup ( $S$, . ) is Intra regular i.e., $x a^{2} y=a$

(or) $\mathrm{ya}^{2} \mathrm{x}=\mathrm{a}$

1.2. Definition: A semi group (S..) is said to be H. Semigroup if

1. $\mathrm{x}^{2}=\mathrm{y}=\mathrm{y}^{2} \Rightarrow \mathrm{x}=\mathrm{y}$

2. If $\mathrm{x}, \mathrm{y} \in \mathrm{S}, \mathrm{u}, \mathrm{v}, \in \mathrm{S}$ and a positive integer $\mathrm{n}$ s.t. $\mathrm{x}^{\mathrm{n}}=\mathrm{uy}$ and $\mathrm{y}^{\mathrm{n}}=\mathrm{vx}$

1.3 .Definition: A semi group (S..) is said to be $\Pi$ - Regular. If positive integer

$\mathrm{a}^{\mathrm{n}}=\mathrm{a}^{\mathrm{n}} \mathrm{xa}^{\mathrm{n}} \forall \mathrm{a}, \mathrm{x} \in \mathrm{S}$ and $\mathrm{n}$ is any

1.4. Definition: A semi group (S..) is said to be left $\Pi$ - inverse semigroup if it is $\Pi$ - regular and a $=$ axa $=$ aya $\Rightarrow$ ax $=$ ay for all $\mathrm{a}, \mathrm{x}, \mathrm{y} \in \mathrm{S}(\mathrm{xa}=\mathrm{ya})$

1.5. Theorem: Every externally commutative right zero semigroup is right regular iff it is intra regular.

Proof: Given that ( $S$,.) is externally commutative semi group $\Rightarrow a x b=b x a$ if $a, b, x \in S$

Let $(\mathrm{S}$, .) be right regular

Now $\mathrm{a}^{2} \mathrm{x}=\mathrm{a}$

$\mathrm{ya}^{2} \mathrm{x}=\mathrm{ya}$ 
$\mathrm{ya}^{2} \mathrm{x}=\mathrm{a} \quad$ (right zero semi group)

Conversely Let $(\mathrm{S},$.$) is intra - regular semi group$

i.e. $x a^{2} y=a$

xa. ay $=\mathrm{a}$

a.ay $=\mathrm{a} \quad$ (right zero semi group)

$a^{2} y=a$

1.6. Theorem: If (S..) is a H-semigroup then weakly cancellative law holds in H-semigroup.

Proof: Let $(\mathrm{S}$, .) be a H-semi group

$x^{2}=x y=y^{2} \Rightarrow x=y$

If $\mathrm{x}, \mathrm{y} \in \mathrm{S}, \mathrm{u}, \mathrm{v}, \in \mathrm{S}$ and a positive integer $\mathrm{n}$ s.t. $\mathrm{x}^{\mathrm{n}}=\mathrm{uy}$ and $\mathrm{y}^{\mathrm{n}}=\mathrm{vx}$

To show that it is weakly cancellative .

i.e. $u x=u y, x v=y v \Rightarrow x=y$

Let $u x=u y, x v=y v$

$$
\begin{aligned}
& \text { Now } x^{n+1}=x^{n} \cdot x \quad y^{n+1}=y^{n} \cdot y \\
& \text { = (uy) } \mathrm{x} \quad=\mathrm{vx} \cdot \mathrm{y} \\
& =\mathrm{uxy} \quad=\mathrm{vy} \cdot \mathrm{x} \\
& \text { = u y y } \quad=y v \cdot x \\
& =x^{\mathrm{n}} \cdot \mathrm{y} \quad=\mathrm{xv} \cdot \mathrm{x} \\
& =y^{n} \cdot x
\end{aligned}
$$

For, $\mathrm{n}>1$

$$
\begin{aligned}
x^{2 n-2} \cdot x y & =x^{n-2} \cdot x^{n+1} \cdot y \\
& =x^{n-2} x^{n} y \quad\left(\because x^{n+1}=x^{n y}\right) \\
& =x^{2 n-2} y^{2}=\left(x^{n-1} y\right)^{2}
\end{aligned}
$$

Similarly,

$$
\begin{aligned}
x^{2 n-2} \cdot x y & =x^{n-1} \cdot x^{n} \cdot y \\
& =x^{n-1} \cdot x^{n+1} \quad\left(\because x^{n+1}=x^{n y}\right) \\
& =x^{2 n} \\
\Rightarrow\left(x^{n-1} \cdot y\right)^{2}= & \left(x^{n}\right)^{2}=x^{n} \cdot\left(x^{n-1} y\right)
\end{aligned}
$$

After (n-1) steps, $x^{2}=x y$

$$
y^{2}=x y
$$

$\because x^{2}=x y=y^{2} \Rightarrow x=y$

Hence $(S,$.$) is weakly cancellative$

1.7. Theorem : If $(\mathrm{S},$.$) is externally commutative semigroup then every inverse semigroup is \mu$-inverse semigroup.

Proof: Given that $(S,$.$) is externally commutative semigroup. i.e., a x b=b x a$ or $a y b=$ bya

Let $(\mathrm{S}$, .) be an inverse semigroup

To prove that $\mathrm{baxc}=\mathrm{bc}$ and byac $=\mathrm{bc}$

Let,

$$
\text { baxc }=b(a x b) x c \quad(\because x b x=x)
$$


$=\mathrm{b}(\mathrm{bxa}) \mathrm{xc} \quad(\because \mathrm{S}$ is externally commutative $)$

$$
\begin{array}{rlr} 
& =b b(x a x) c \quad(\because x a x=x) \\
& =b b x c \\
& =b x b c \quad(\text { commutative }) \\
\text { baxc } & =b c \quad(\because b=b x b)
\end{array}
$$

Similarly,

$$
\begin{aligned}
& \text { byac }=\text { bybyac } \quad(\because y=\text { yby }) \\
& =\text { by }(\text { bya }) c \\
& =\text { by }(\mathrm{ayb}) \mathrm{c} \quad(\because \mathrm{S} \text { is externally commutative }) \\
& =\mathrm{b} \text { (yay)bc } \\
& =\text { bybc }(\because \text { yay }=\text { y }) \\
& =\mathrm{bc} \quad(\because \mathrm{byb}=\mathrm{b})
\end{aligned}
$$

Hence $\mathrm{S}$ is $\mu$-Inverse semigroup.

1.8. Lemma: Every cancellative GC-commutative is commutative.

Proof: Let (S, .) be cancellative GC-commutative.

i.e., $x^{2} y x=x^{2} x^{2}$ for all $x, y$ in $S$.

$$
\begin{aligned}
& \Rightarrow \mathrm{xx} y \mathrm{x}=\mathrm{xyxx} \\
& \Rightarrow \mathrm{xy} \quad=\mathrm{yx} \quad \text { (since } \mathrm{S} \text { is cancellative) }
\end{aligned}
$$

Therefore, $\mathrm{S}$ is commutative.

1.9. Lemma: Every commutative semigroup is GC-commutative.

Proof : Let $(\mathrm{S},$.$) be a commutative semigroup.$

Now,

$\mathrm{xy} \quad=\mathrm{yx}$ for all $\mathrm{x}, \mathrm{y}$ in $\mathrm{S}$.

$$
\begin{array}{lll}
\Rightarrow & x \cdot x y \quad=x \cdot y x \\
\Rightarrow & x \cdot x y \cdot x=x \cdot y x \cdot x \\
\Rightarrow & x^{2} y x \quad=x y x^{2}
\end{array}
$$

Hence, $\mathrm{S}$ is GC-commutative.

1.10. Note: But every GC-commutative is not commutative.

1.11. Theorem: Every permutable semigroup is externally commutative.

Proof: Given that $(\mathrm{S},$.$) is permutable semigroup.$

i.e., $a x b=x a b=a b c \forall a, b, x \in S$

To prove that $(\mathrm{S},$.$) is externally commutative.$

$$
\begin{aligned}
\text { Consider } \mathrm{axb} & =\mathrm{abx} & & \text { (right permutable) } \\
& =\mathrm{bax} & & \text { (left permutable) } \\
& =\mathrm{bxa} & & \text { (right permutable) }
\end{aligned}
$$

$\because(\mathrm{S},$.$) is externally commutative.$

1.12. Theorem: Every permutable semigroup is GC-commutative semigroup.

Proof: Let $(\mathrm{S}$, .) be permutable semigroup.

i.e $x y z=x z y=y x z \forall x, y, z \in S$ 
To prove it is GC-commutative

$$
\text { Consider } \quad \begin{aligned}
\mathrm{x}^{2} \mathrm{yx} & =\mathrm{yx}^{2} \mathrm{x} \\
& =\mathrm{yxx}^{2} \\
& =\mathrm{xyx}^{2}
\end{aligned}
$$

Hence $(\mathrm{S},$.$) is GC-commutative.$

1.13. Theorem: Every commutative left(right) regular semigroup is regular.

Proof: Given that $(\mathrm{S},$.$) is left(right) regular.$

\begin{tabular}{|c|c|c|}
\hline$(x a) a$ & $=\mathrm{a}$ & $a(a x)$ \\
\hline axa & $=\mathrm{a}$ & axa \\
\hline
\end{tabular}

i.e $\mathrm{xa}^{2}=\mathrm{a}\left(\mathrm{a}^{2} \mathrm{x}=\mathrm{a}\right) \quad \forall \mathrm{a}, \mathrm{x} \in \mathrm{S}$

Let $\mathrm{xa}^{2}=\mathrm{a} \quad$ similarly $\mathrm{a}^{2} \mathrm{x}=\mathrm{a}$

Hence, $(S,$.$) is regular.$

1.14. Theorem: Every commutative left regular semigroup is left $\pi$-inverse semigroup.

Proof: Given that $(\mathrm{S},$.$) is commutative and left regular semigroup.$

$$
\text { i.e., } x y=y x \text { and } x a^{2}=a \forall a, x, y \in S
$$

To prove that $(\mathrm{S},$.$) is left \pi$-inverse semigroup.

For this first we have to prove that (1) $\mathrm{S}$ is $\pi$-regular

$$
\text { (2) } a=a x a=a y a \Rightarrow a x=a y
$$

First we need to show that it is $\pi$-regular.

For any $n$ is positive integer i.e., $a^{n}=a^{n} x a^{n} \forall a, x \in S$.

$$
\begin{aligned}
\text { Let } a^{n}= & a^{n-1} \cdot a \\
& =a^{n-1} \cdot x a^{2} \\
& =a^{n-1} \cdot(x a) a \\
& =a^{n-1} \cdot a x a \\
& =a^{n} \cdot x \cdot a \\
& =a^{n} \cdot x \cdot x a^{2} \\
& =a^{n} x \times a a \\
& =a^{n} x x\left(x a^{2} x\right) a^{2} \\
& =a^{n} x x a^{2} \cdot a^{2} \\
& =a^{n} x\left(a^{2} x a^{2}\right) \\
& \left.=a^{n} \cdot x \cdot a^{2} \quad \text { (S is commutative }\right)
\end{aligned}
$$

If we continue like this,

$$
a^{n}=a^{n} \cdot x \cdot a^{n}
$$

Hence $(\mathrm{S},$.$) is \pi$-regular.

(2) Now let,

$$
\begin{aligned}
\text { axa } & =\text { aya } \\
\Rightarrow \text { axax } & =\text { ayax } \\
\Rightarrow a x & =\text { yaax }[\because \text { axa }=a]
\end{aligned}
$$




$$
=\text { yaxa }=\text { ya }=\text { ay }
$$

Hence $(S,$.$) is \pi$-Inverse semigroup.

1.15. Theorem: If $(S,$.$) is commutative weakly balanced semigroup then every left(right) regular semigroup is$ weakly separative, quasi separative and separative.

Proof: Given $(\mathrm{S},$.$) is commutative weakly balanced semigroup.$

i.e., $x a=y a, b x=b y$

If $(\mathrm{S}$,$) is left (right) regular$

$\mathrm{xa}^{2}=\mathrm{a}\left(\mathrm{a}^{2} \mathrm{x}=\mathrm{a}\right)$

$\mathrm{yb}^{2}=\mathrm{b}\left(\mathrm{b}^{2} \mathrm{y}=\mathrm{b}\right) \quad \forall \mathrm{a}, \mathrm{b}, \mathrm{x}, \mathrm{y} \in \mathrm{S}$

To prove that $(S,$.$) is weakly separative$

i.e., $a^{2}=a b=b^{2} \Rightarrow a=b$

$\begin{array}{rlrl}\text { Let } \quad & \mathrm{a}^{2}=\mathrm{ab} & \text { Similarly, } & \mathrm{b}^{2}=\mathrm{ab} \\ \mathrm{xa}^{2}=\mathrm{xab} & & \mathrm{yb}^{2}=\mathrm{yab} \\ = & \mathrm{yab}--------(1) & \mathrm{yb}^{2}=\mathrm{yab}\end{array}$

From (1) and (2) $\mathrm{xa}^{2}=\mathrm{yab}=\mathrm{yb}^{2}$

$$
\begin{aligned}
& \Rightarrow \mathrm{xa}^{2}=\mathrm{yb}^{2} \\
& \Rightarrow \mathrm{a}=\mathrm{b}
\end{aligned}
$$

Therefore, $(S,$.$) is weakly separative.$

To show that $(S,$.$) is quasi separative$

i.e $a^{2}=a b=b a=b^{2} \Rightarrow a=b$

Now $b^{2}=b a$

$$
\begin{aligned}
\Rightarrow \mathrm{yb}^{2} \quad & \text { yba } \\
& =\text { bya } \\
& =\text { bxa } \\
& =x b a \\
& =x(a b) \\
& =x^{2} \\
\Rightarrow b= & \\
\Rightarrow(S, .) & \text { is quasi separative }
\end{aligned}
$$

To show that $\mathrm{S}$ is separative.

$$
\begin{aligned}
& \text { i.e } \quad a^{2}=a b \quad a^{2}=b a \\
& \checkmark \\
& \mathrm{a}=\mathrm{b} \text { and } \Rightarrow \mathrm{a}=\mathrm{b} \\
& \mathrm{b}^{2}=\mathrm{ba} \quad \mathrm{b}^{2}=\mathrm{ab} \\
& \text { Let } \mathrm{a}^{2}=\mathrm{ab} \quad \text { and } \quad \mathrm{b}^{2}=\mathrm{ba} \\
& \Rightarrow \mathrm{xa}^{2}=\mathrm{xab} \quad \text { and } \mathrm{yb}^{2}=\mathrm{yba} \\
& \text { = yab ------(1) =yab }
\end{aligned}
$$


From (1) and (2) $\mathrm{xa}^{2}=\mathrm{yb}^{2}$

$$
\Rightarrow \mathrm{a}=\mathrm{b}
$$

Similarly, Let $\mathrm{a}^{2}=\mathrm{ba}$ and $\quad b^{2}=a b$

$$
\mathrm{xa}^{2}=\mathrm{xba}-----(3) \quad \text { and } \quad \mathrm{yb}^{2}=\mathrm{yab}
$$

From (3) and (4) $x a^{2}=y b^{2} \Rightarrow a=b$

Hence $(\mathrm{S},$.$) is separative.$

1.16. Theorem: Every completely regular semigroup is permutable.

Proof: Given that $(\mathrm{S},$.$) is completely regular.$

i.e., $a x a=a, x a=a x$

To prove that $\mathrm{S}$ is permutable

i.e $a x b=x a b=a b x$ for any $a, b, x$ in $S$

$(\because a=a x a)$

$=(\mathrm{ax})(\mathrm{ax}) \mathrm{b}$

$=x(a x a) b$

$(\mathrm{xa}=\mathrm{ax})$

$=\mathrm{xab}$

$(\because \operatorname{axa}=a)$

Similarly, $a x b=a x b x b$

$$
\begin{array}{ll}
=a(b x b) x & \\
=a b x & (b x b=b)
\end{array}
$$

Hence $(S$, .) is permutable.

1.17. Theorem: If $(S,$.$) is completely regular semigroup. Then it is weakly separative.$

Proof: Given that $(\mathrm{S},$.$) is completely regular$

To prove that $(\mathrm{S},$.$) is weakly separative$

$$
\begin{aligned}
& \text { i.e } a^{2}=a b=b^{2} \Rightarrow a=b \\
& \text { Now } a^{2}=a b \text { and } \quad b^{2}=a b \\
& \Rightarrow \quad a \cdot a=a b \text { and } \quad b \cdot b=a b \\
& \Rightarrow \quad \text { aax }=a b x \quad \text { and } \quad b \cdot b x=a b x \\
& \Rightarrow \quad a x a=b^{2} x \quad \text { and } \quad b x b=a^{2} x \\
& \Rightarrow \quad \text { axa }=\text { bbx } \quad \text { and } \quad b x b=a a x \\
& \Rightarrow \quad a x a=b x b \quad \text { and } \quad b x b=a x a \\
& \Rightarrow \quad \mathrm{a}=\mathrm{b} \quad \text { and } \quad \mathrm{b}=\mathrm{a}
\end{aligned}
$$

Hence $(S,$.$) is weakly separative$

\section{References}

Howie, J.M "Introduction to semi group theory" academic press. London, 1976 Sreenivasulu Reddy,P. \&Some studies on Regular semigroups (Thesis) and Shobhalatha,G. Tamura, T. and Kimura, N., On decomposition of commutative semigroups. Kodai math Sem. Rep. 109-112 (1954) 